\title{
Computer-Aided Arrhenius-Evaluation of Kinetic Data
}

\author{
P. Hugo, J. Leonhardt, and S. Wagner \\ Institut für Technische Chemie, Technical University Berlin, D-10623 Berlin \\ Z. Naturforsch. 50 a, 549-554(1995); received December 16, 1994 \\ Dedicated to Prof. E. Wicke on the occation of his $80^{\text {th }}$ birthday
}

\begin{abstract}
A new evaluation method is presented to analyse the temperature dependance of reactions with rates that can be separated into a temperature dependent and a concentration dependent part. The proposed method allows to evaluate isothermal, adiabatic, isoperibolic and temperature programmed measurements in an unified manner. To determine the activation energy an Arrhenius law but no explicite kinetic model has to be assumed. The application of the method is demonstrated using experimental data of different reactions from former investigations.
\end{abstract}

Key words: Kinetic modeling, non-isothermal measurements, reaction kinetic, Arrhenius law, Differential Scanning Calorimetry

\section{Problems of kinetic modeling}

There are two problems in kinetic modeling. The experimental difficulties connected with the determination of precise data are the first problem. The second problem is the model formulation and the fitting of the kinetic parameters. Several studies of kinetic model discrimination and parameter estimation have shown that the same set of experimental data often can be described by different models and different parameters with the same accuracy [1], [2]. A detailed study of this problem was presented by Berty [3]. Simulated data of the catalytic methanol synthesis were evaluated by 19 groups from eight countries. The diversity of the models proposed is astonishing.

Of course, the heterogeneously catalyzed methanol synthesis is not a simple example due to the reversibility of this reaction. However even for a simple, irreversible homogeneous reaction it was shown in [4], that the measured kinetic data can be described by several sets of kinetic parameters with the same accuracy.

During the last two decades a large number of investigations was published discussing the application of computers for kinetic modeling. A review of the state of the art in 1983 was presented by Côme [5]. One serious problem is the selection of a proper model

Reprint requests to Prof. Dr. P. Hugo. that is on the one hand sufficiently general to describe the measured data and on the other hand contain not more parameters than necessary. However, the main question seems to be whether the problem of kinetic modeling is mainly a mathematical problem or a question of chemical understanding.

A kinetic model justified by chemical understanding can only be formulated for homogeneous elementary reactions in the gas phase or in solutions at infinite dilution. So, any kinetic model describing chemical reactions under technical conditions will be more or less empirical. In the present paper it will be shown that for several types of reaction a computer aided Arrhenius evaluation can be performed without additional model assumptions.

The methods presented are discussed for the example of homogeneous liquid-phase reactions. However the same principles of evaluation can also be used for heterogeneous catalytic reactions.

\section{The temperature dependence of reaction rate}

Generally the experimental determination of kinetic data is performed under isothermal conditions. Then the first step of a kinetic analysis is the fitting of a rate law to such data. In simple cases this may be a rate law of $n$-th order. But mostly, more complicated rate laws have to be used. In the second step, the rate constants determined at different temperatures are described by an Arrhenius law.

0932-0784 / 95 / 0600-0549 \$ 06.00 C - Verlag der Zeitschrift für Naturforschung, D-72072 Tübinæen 


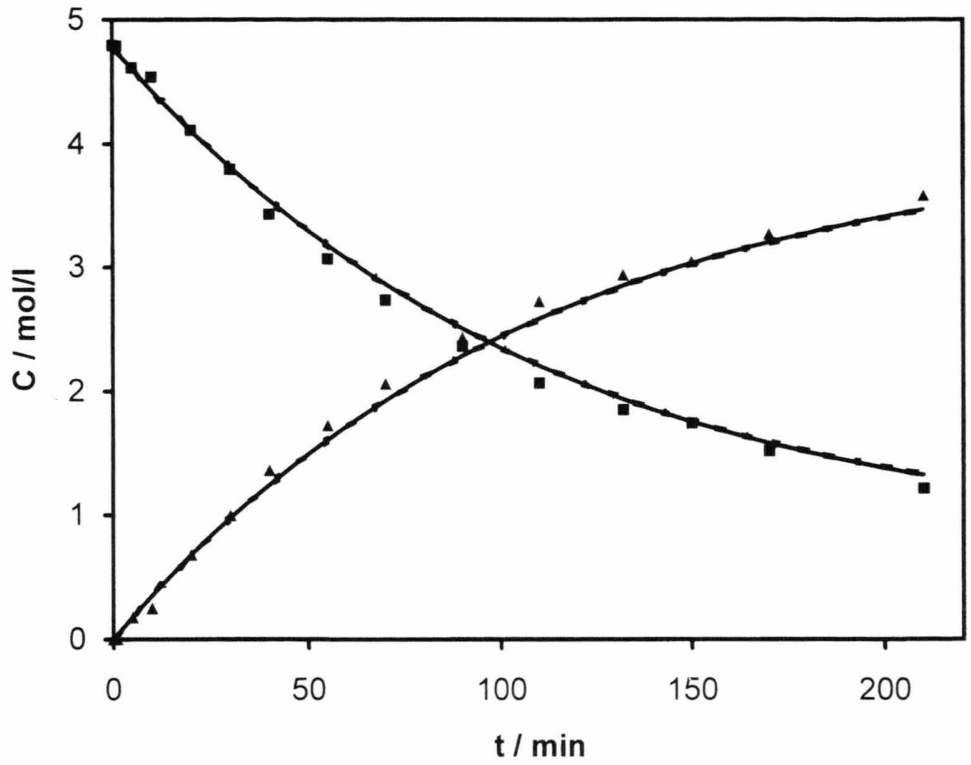

Fig. 1. Concentration courses of catalyzed alcoholysis of acetic anhydrid by benzylalcohol at $35^{\circ} \mathrm{C} ;-$ calculated with $b=f(T), \cdots$ calculated with $b=$ const., $\Delta$ experimental ester concentration, - experimental alcohol concentration.
Thermokinetic measurements are often performed under nonisothermal conditons. Evaluating such types of measurements, other evaluation principles were found to be more successful [6]. In the first step the kinetic data are fitted to an Arrhenius law to obtain the activation energy. Taking this result all data now can be transformed to equivalent isothermal data at a reference temperature $T_{\mathrm{R}}$. These data are fitted to a proper rate law in the second step.

This way of evaluation may not only be successful in nonisothermal measurements but also of advantage in evaluating isothermal measurements at different temperatures. The reason is that under certain conditions an Arrhenius analysis can be performed without further assumptions concerning the rate law. First the conditions for such an Arrhenius evaluation have to be defined.

One condition is that only one reaction takes place which can be described by a stoichiometric equation

$$
\nu_{1} A_{1}+\nu_{2} A_{2}=\nu_{3} A_{3}+\ldots
$$

If the reaction is irreversible, the rate law often can be separated into a temperature dependent Arrhenius part and a concentration dependent part at a reference temperature $T_{\mathrm{R}}$ :

$$
r\left(c_{i}, T\right)=\exp \left[\frac{E}{R}\left(\frac{1}{T_{\mathrm{R}}}-\frac{1}{T}\right)\right] \cdot r\left(c_{i}, T_{\mathrm{R}}\right) .
$$

In some cases, (2) may be an approximation. As an experimental example we will discuss the acid catalyzed alcoholysis of acetic anhydride $A_{1}$ by benzylalcohol $A_{2}$ in a batch reactor. The following rate law was determined previously [7] by isothermal measurements.

$$
r\left(c_{i}, T\right)=k(T) \cdot \frac{c_{\mathrm{K}} \cdot c_{1}^{2} c_{2}}{1+b(T) \cdot c_{1} c_{2}} .
$$

$c_{\mathrm{K}}$ is the concentration of $\mathrm{p}$-toluene sulfonic acid as catalyst. The rate law contains two temperature dependent constants:

$$
\begin{array}{ll}
k(T)=3.0 \cdot 10^{9} \cdot \exp \left(\frac{-60400}{R T}\right) & 1^{3} \mathrm{~mol}^{-3} \mathrm{~s}^{-1} \\
b(T)=1.6 \cdot 10^{-3} \cdot \exp \left(\frac{14700}{R T}\right) & 1^{2} \mathrm{~mol}^{-2}
\end{array}
$$

In the case of the rate law given by (3), a separated one according to (2) is not possible. However, eq.(3) was not derived from a reaction mechanism but verified by fitting to the experimental data. Any other rate law which fits the data with the same acurracy might be used as well. Therefore, the original data of Pham [7] were reconsidered by the method described in Sect. 3 below. The result is a rate law according to (3) but with a temperature independent value of $b$ :

$$
\begin{array}{lll}
k(T) & =8.6 \cdot 10^{11} \cdot \exp \left(\frac{-75100}{R T}\right) & \mathrm{l}^{3} \mathrm{~mol}^{-3} \mathrm{~s}^{-1} \\
b & =0.4 & \mathrm{l}^{2} \mathrm{~mol}^{-2}
\end{array}
$$




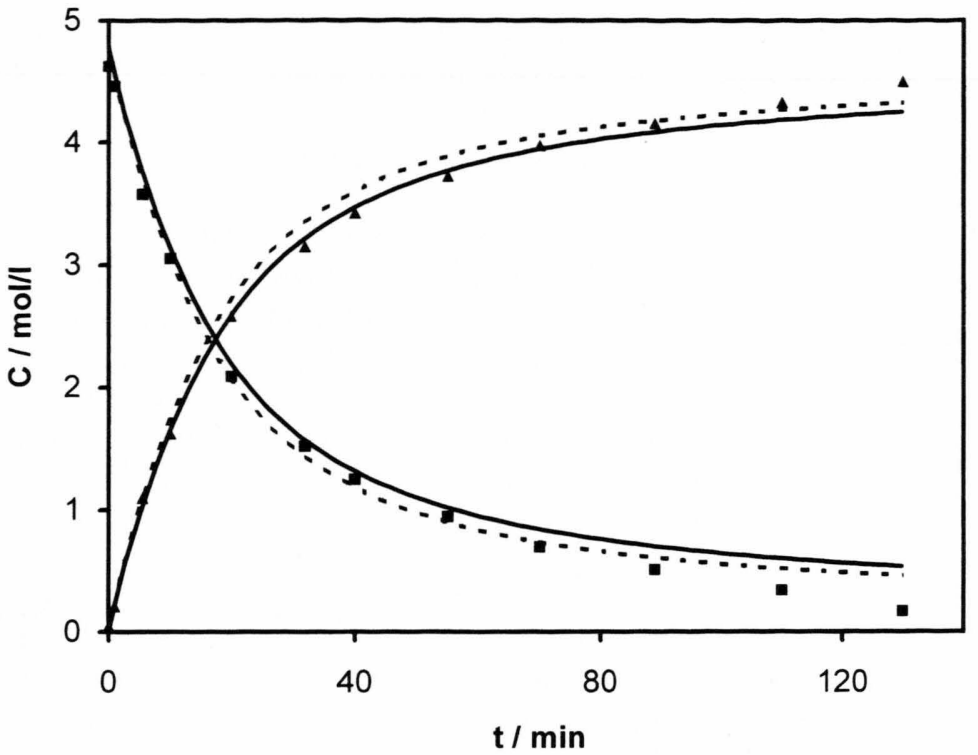

Fig. 2. Concentration courses of catalyzed alcoholysis of acetic anhydrid by benzylalcohol at $55^{\circ} \mathrm{C}$; - calculated with $b=f(T), \cdots$ calculated with $b=$ const., $\Delta$ experimental ester concentration, - experimental alcohol concentration.
Now the rate law can be represented in the type given by (2). Figures 1 and 2 show a comparison of the theoretical results with the experimental data. The experimental data were determined at the lowest and the highest measured temperature with a nearly stoichiometric initial composition of the reactants. In this case there is the largest influence of the b-factor on the reaction rate.

This example shows that even kinetic rate laws with more than one temperature dependent parameter can be expressed in a simplified manner with (2). Problems may arise for heterogeneous catalytic reactions if the activation energy and the heat of chemisorption are of the same order of magnitude. Also reversible reactions can cause problems due to the temperature dependence of the equilibrium composition.

If the investigated reaction is reversible, the chemical equilibrium has to be taken into account. The equilibrium of a liquid system can be described by concentrations $c_{i, \text { eq }}$.

$$
K_{c}(T)=\prod_{i} c_{i, \mathrm{eq}}^{\nu_{i}}
$$

In most cases, $K_{c}$ can be calculated from thermodynamic data. In concentrated solutions these calculations are complicated and not always reliable. It might be often easier to measure the chemical equilibrium using the same experimental set up that is applied to kinetic measurements.
The microscopic reversibility postulates that the ratio of backward reaction rate $r_{b}$ to forward rate $r_{f}$ is

$$
\left(\frac{r_{b}}{r_{f}}\right)_{\mathrm{eq}}^{m}=K_{c}^{-1} \cdot \prod_{i} c_{i}^{\nu_{i}}
$$

In most cases holds $m=1$. Consequently, for practical purposes the rate law of reversible reactions can be described by

$$
r_{\mathrm{rev}}\left(c_{i}, T\right)=r_{f}\left(c_{i}, T\right) \cdot\left(1-K_{c}(T)^{-1} \cdot \prod_{i} c_{i}^{\nu_{i}}\right)
$$

In this equation, $c_{i}$ are the actual concentrations and $r_{f}$ the forward reaction rate described by (2). Provided $K_{c}(T)$ is available, the temperature dependence of reversible reactions can be described from (2) and (8).

\section{The principles of the general Arrhenius evaluation}

We assume that the kinetic measurements are performed in a closed system. The mass balance of a component $A_{i}$ than can be written in the form

$$
\frac{1}{\nu_{i}} \cdot \frac{\mathrm{d} c_{i}}{\mathrm{~d} t}=r\left(c_{i}, T\right)
$$


The concentrations $c_{i}$ can be expressed by the initial concentration $c_{i 0}$ and the conversion $X$ of the key component $A_{1}$ :

$$
c_{i}=c_{i 0}-\frac{\nu_{i}}{\nu_{1}} \cdot c_{10} X .
$$

Thus, the rate law is expressed in terms of conversion and temperature. With these variables the mass balance holds

$$
\frac{\mathrm{d} X}{\mathrm{~d} t}=\frac{\left(-\nu_{1}\right) \cdot r\left(c_{i 0}, X, T\right)}{c_{10}} .
$$

With (2) and (8) the rate law can be separated:

$$
\begin{aligned}
r\left(c_{i 0}, X, T\right)=(1- & \left.K_{c}(T)^{-1} \cdot \prod_{i} c_{i}^{\nu_{i}}\right) \\
& \cdot r_{f}\left(c_{i 0}, X, T_{\mathrm{R}}\right) \\
& \cdot \exp \left[\frac{E}{R}\left(\frac{1}{T_{R}}-\frac{1}{T}\right)\right] .
\end{aligned}
$$

It is expedient to define an equivalent reaction time $h$ at the reference temperature $T_{\mathrm{R}}$ by the integral

$$
\begin{aligned}
h(t)=\int_{0}^{t}\left(1-K_{c}(T)^{-1} \cdot \prod_{i} c_{i}^{\nu_{i}}\right) \\
\cdot \exp \left[\frac{E}{R}\left(\frac{1}{T_{\mathrm{R}}}-\frac{1}{T}\right)\right] d t .
\end{aligned}
$$

Now the mass balance (9) can be rearranged to a balance at the reference temperature containing only the conversion $X$ as variable:

$$
\frac{d X}{d h}=\frac{\left(-\nu_{1}\right) \cdot r_{f}\left(c_{i 0}, X, T_{\mathrm{R}}\right)}{c_{10}} .
$$

Equations (13) and (14) are the basis for a general, computer aided Arrhenius evaluation of measured kinetic data. According to (14) a set of kinetic measurements with the same initial concentrations but different temperature conditions reveals the same course of $X(h)$, independent of the special type of rate law $r\left(c_{i}, X, T\right)$. As the conversion $X$ can be calculated directly from the measured data, the main task is how to calculate the equivalent reaction time $h$ from (13). For an irreversible reaction $\left(K_{c} \rightarrow \infty\right)$ the only unknown value in (13) is the activation energy $E$. The temperature-time course can be usually determined experimentally for each run as a close sequence of points. So, $h$ of (13) can be obtained by numerical integration for an assumed value of $E$. The correct value of $E$ is found if all curves $X(h)$ have the same course. A corresponding computer program can easily be developed.

For reversible reactions, $K_{c}(T)$ has to be determined separately. The concentrations $c_{i}$ can be calculated as functions of the conversion using (10). However, to perform a numerical integration of (13) a sufficient amount of data $X(t)$ for each measurement has to be available. A method to fit a set of measured data points $X(t)$ to an empirical function was described in [4]. In this way, for reversible reactions the equivalent reaction time $h$ can be calculated numerically for any value of $E$. The correct value of $E$ then is obtained in the same way as for irreversible reactions.

When the activation energy has been determined, all other parameters of the rate law can be obtained using (14). This proposed "equivalent" isothermal evaluation can be applied to all conventional methods to determine kinetic models described in [5]. To evaluate the experimental data of the subsequent sections, a differential method is used, as described in [4]. As the purpose of the present paper is the description of the general Arrhenius evaluation, the further mathematical description of the rate laws will not be discussed in detail.

\section{Experimental Section}

\subsection{Irreversible reactions}

Based on the evalution method presented above, the computer program UVTHERK was developed. The program was tested with several simulated data. The agreement between the obtained and prescribed parameters was very good.

The Arrhenius evaluation was first tested using experimental data of the simple reaction of phenylisocyanate with 2-butanole. The results in [6] agree with parameters described in the literature. Two other investigated reactions were the decomposition of dimethyl-2,2'-azobisisobutyrate and the curing reaction of the two-component adhesive UHUEndfest 300 measured with a power-compensating DSC (Perkin-Elmer, model DSC-7). The results and the experimental conditions are published in [8]. In [8] only one characteristic point of each measurement was used to estimate the activation energy.

The decomposition reaction mentioned above is another example for a simple reaction. The time $t$, temperature $T$ and conversion $X$ obtained from the 


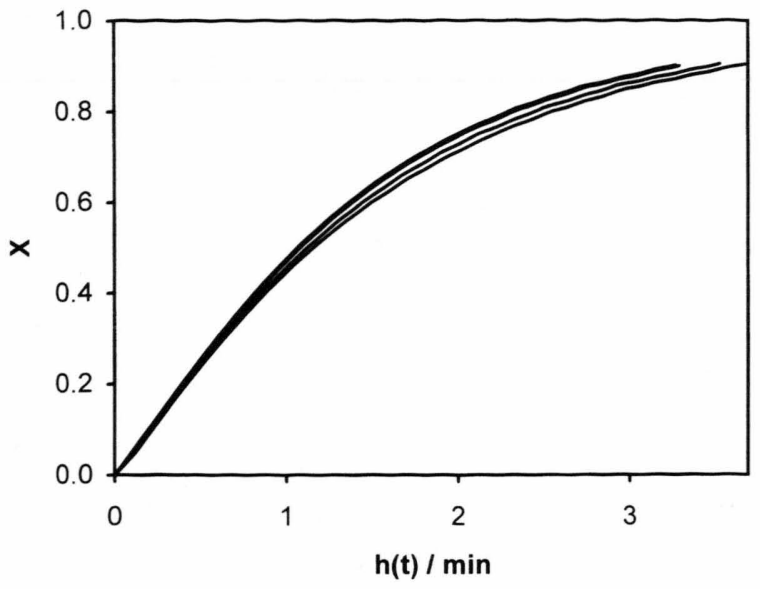

Fig. 3. $X$ vs. $h(t)$ curves of the decomposition of dimethyl2,2-azobisisobutyrate calculated with $E / R=14500 \mathrm{~K}$.

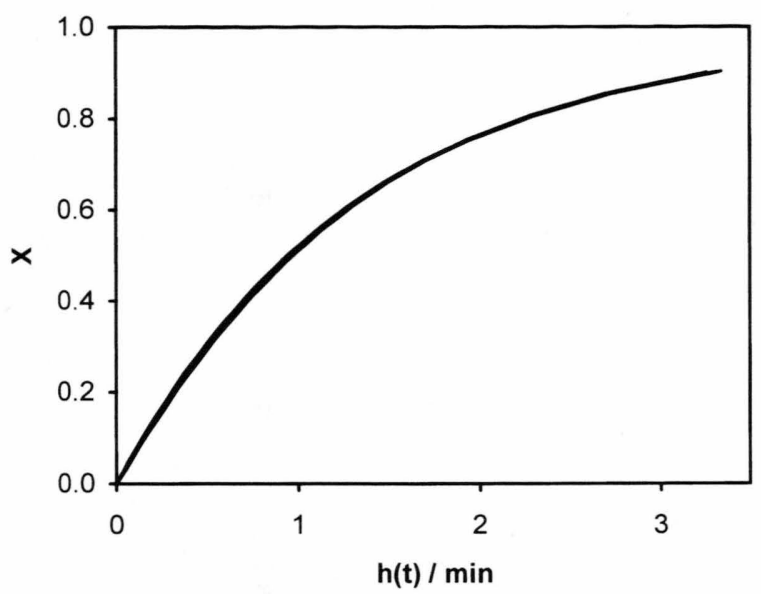

Fig. 4. $X$ vs. $h(t)$ curves of the decomposition of dimethyl2,2-azobisisobutyrate calculated with $E / R=15800 \mathrm{~K}$.

measurements form the base of the Arrhenius evaluation. Figure 3 shows the determined curves $X(h)$ for an estimated value $E / R=14500 \mathrm{~K}$. Because of the different courses of the curves the value of $E / R$ has to be optimized with respect to the objective function to transform all curves $X(h)$ into one curve. The visually optimized value is $E / R=(15800 \pm 300) \mathrm{K}$ (Figure 4). In this range all courses of $X(h)$ visually do not differ from each other.

This optimized activation energy is in accordance with the literature [9]. Moreover, evaluating the equivalent isothermal data of Figure 4, a rate law of first order is obtained in agreement with [9].

The second example is the more complex curing reaction of the two-component adhesive UHU-

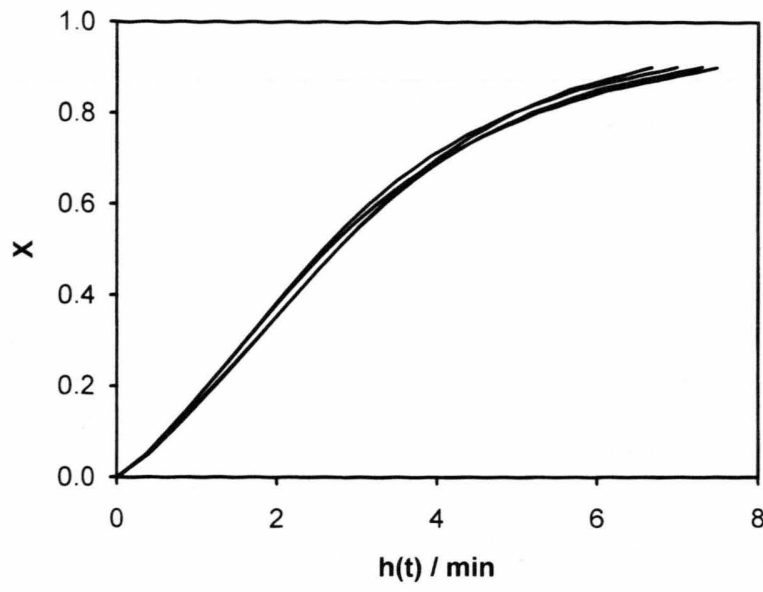

Fig. 5. $X$ vs. $h(t)$ curves of the curing reaction of a twocomponent adhesive calculated with $E / R=6600 \mathrm{~K}$.

Endfest 300. The best achievable agreement of the $X(h)$ curves for $E / R=6600 \mathrm{~K}$ is shown in Figure 5. Comparable results are obtained with values in the range from $E / R=6500 \mathrm{~K}$ to $E / R=6700 \mathrm{~K}$, indicating the range of uncertainty.

The subsequent evaluation revealed an autocatalytical behaviour of this reaction. Of course in this case the description by a simple Arrhenius law is only an approximation. Actually the complex curing reaction can not be described by one simple stochiometric equation (1).

\subsection{Reversible reactions}

The esterfication of dicarbonic acid anhydrid with an alcohol is a two step reaction. The first step of the reaction of phthalic acid anhydrid with benzyl alcohol was investigated in [10]. It was found that this reaction forms the monoester in an autocatalytic reversible reaction. In detailed evaluation the consecutive reaction of the monoester to the diester has to be considered. This can not be done by the method presented here. However, to get some realistic data for testing the new evaluation method for a reversible reaction, the kinetic data obtained in [10] were used for simulation of isothermal reaction pathes at different temperatures $\left(70{ }^{\circ} \mathrm{C}, 100{ }^{\circ} \mathrm{C}, 130{ }^{\circ} \mathrm{C}, 160{ }^{\circ} \mathrm{C}\right)$, neglecting the consecutive reaction. The main interest in the evaluation was the temperature dependence of the reaction rate, so the details of the kinetics are again not discussed here. The temperature dependence of the forward reaction rate obtained by Rau [10] was $E / R=6660 \mathrm{~K}$. 


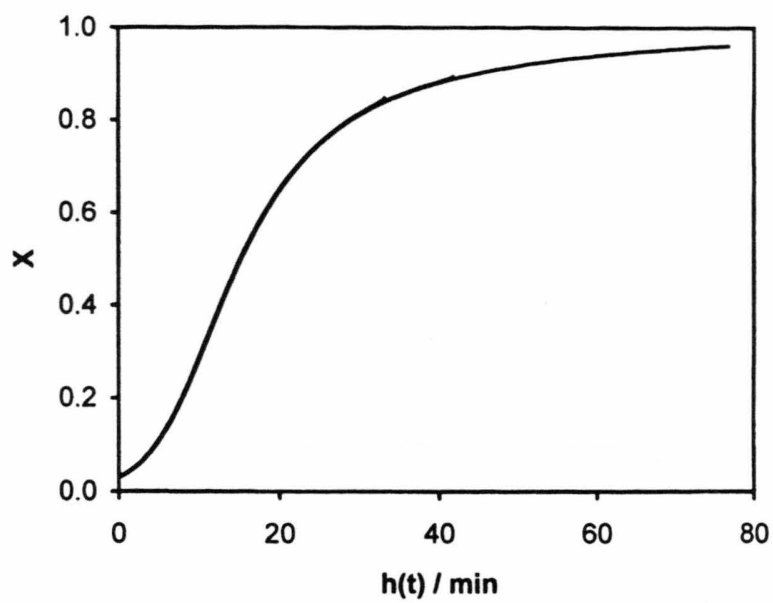

Fig. 6. Arrhenius-Evaluation of a reversible reaction with $-\Delta_{\mathrm{R}} H / R=3900 \mathrm{~K}$.

The temperature dependence of the equilibrium corresponds to $\Delta H_{R} / R=-3900 \mathrm{~K}$. With simulated data and the given equilibrium constant and its temperature dependence the value of $E / R$ is also obtained by a new evaluation. This evaluation is rather sensitive to the temperature dependence of the equilibrium constant. Figure 6 shows the Arrhenius evaluation with the correct values of $E / R$ and $\Delta H_{R} / R$ as used for simulation. Indicating the sensitivity to the temperature dependence of the equilibrium constant, Fig. 7 shows the evaluation with the correct value of $E / R$ but the non-correct value of $\Delta H_{R} / R=-4050 \mathrm{~K}$. As demonstrated in Fig. 7, errors in the determination of the equilibrium constant are of main importance, especially at high conversions close to equilibrium.

This emphasizes that in the case of equilibrium reactions this method requires a precise determination of the equilibrium constant. Otherwise no correct determination of $E / R$ is possible.

[1] L. Petrov, A. Eliyas, and C. Maximov, Ind. Eng. Chem. Research 30, 639 (1991).

[2] L. Biegler, J. Damiano, and G. Blau, AIChE Journal 32, 29 (1986).

[3] J. Berty, Chem. Eng. Prog. 84, 61 (1988).

[4] A. Angelidou and P. Hugo, Chem. Ing. Tech., 62, 741 (1990).

[5] G. Côme, Chem. Kin. 24, 249 (1983).

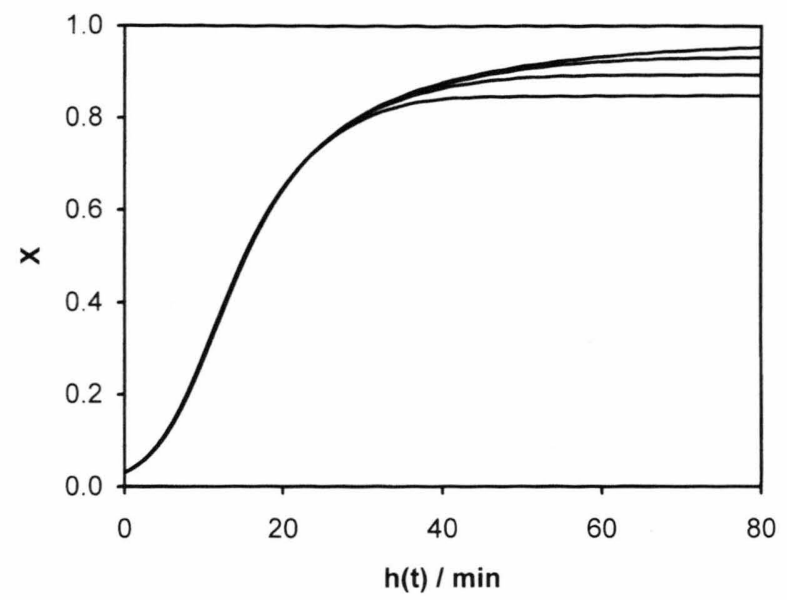

Fig. 7. Arrhenius-Evaluation of a reversible reaction with $-\Delta_{\mathrm{R}} H / R=4050 \mathrm{~K}$.

\section{List of Symbols}

\begin{tabular}{|c|c|c|}
\hline$A_{i}$ & & component $\mathrm{i}$ of reaction \\
\hline$b(T)$ & $\mathrm{l}^{2} \mathrm{~mol}^{-2}$ & \\
\hline$c_{i}$ & $\mathrm{moll}^{-1}$ & concentration of compound $i$ \\
\hline$c_{i 0}$ & $\mathrm{moll}^{-1}$ & $\begin{array}{l}\text { initial concentration } \\
\text { of compound } i\end{array}$ \\
\hline$E$ & $\mathrm{~J} \mathrm{~mol}^{-1}$ & activation energy \\
\hline$h(t)$ & $\mathrm{s}$ & equivalente reaction time \\
\hline$k(T)$ & $\mathrm{l}^{3} \mathrm{~mol}^{-3} \mathrm{~s}^{-1}$ & reaction rate constant in (3) \\
\hline$K_{\mathrm{c}}$ & $\left(\mathrm{moll}^{-1}\right)^{\sum \nu_{i}}$ & equilibrium constant \\
\hline$r$ & $\mathrm{~mol} \mathrm{l}^{-1} \mathrm{~s}^{-1}$ & reaction rate \\
\hline$R$ & $\mathrm{~J} \mathrm{~mol}{ }^{-1} \mathrm{~K}^{-1}$ & gas constant \\
\hline$t$ & $\mathrm{~s}$ & time \\
\hline$T$ & $\mathrm{~K}$ & temperature \\
\hline$T_{\mathrm{R}}$ & $\mathrm{K}$ & reference temperature \\
\hline$X$ & & conversion \\
\hline$\nu_{i}$ & & $\begin{array}{l}\text { stochiometric coefficient } \\
\text { of compound } i\end{array}$ \\
\hline$\Delta H_{\mathrm{R}}$ & $\mathrm{J} \mathrm{mol}^{-1}$ & reaction enthalpy \\
\hline
\end{tabular}

[6] P. Hugo, Chem. Ing. Tech. 65, 1497 (1993).

[7] G. Pham and P. Hugo, Chem. Ing. Tech. 61, 635 (1989).

[8] S. Wagner, P. Hugo, and T. Gnewikow, Thermochim. Acta 225, 153 (1993).

[9] Wako Chemical GmbH, Technical Information of V601 (dimethyl-2,2'-azobisisobutyrate).

[10] C. Rau, Dissertation TU Berlin 1990. 